\title{
Social Vulnerability Subtheme Analysis Improves Perioperative Risk Stratification in Hepatopancreatic Surgery
}

\author{
Hanna E. Labiner ${ }^{1} \cdot$ Madison Hyer ${ }^{1}$. Jordan M. Cloyd ${ }^{1}$. Diamantis I. Tsilimigras ${ }^{1}$. Djhenne Dalmacy ${ }^{1}$. \\ Alessandro Paro ${ }^{1} \cdot$ Timothy M. Pawlik ${ }^{1}$
}

Received: 17 September 2021 / Accepted: 1 January 2022 / Published online: 12 January 2022

(c) The Society for Surgery of the Alimentary Tract 2022

\begin{abstract}
Background There has been increased interest in understanding how social determinants of health (SDH) may affect care both in the medical and surgical setting. We sought to define the impact of various aspects of social vulnerability on the ability of patients to achieve a "textbook outcome" (TO) following hepatopancreatic surgery.

Methods Medicare beneficiaries who underwent hepatopancreatic resection between 2013 and 2017 were identified using the Medicare database. Social vulnerability was defined using the Centers for Disease Control Social Vulnerability Index (SVI), which is comprised of four subthemes: socioeconomic (SE), household composition and disability (HCD), minority status and language (MSL), and housing type and transportation (HTT). TO was defined as the composite endpoint: absence of 90-day mortality or readmission, absence of an extended length of stay (LOS), and no complications during the index admission. Cluster analysis was used to identify vulnerability cohorts, and multivariable logistic regression was utilized to assess the impact of these SVI subthemes on the likelihood to achieve a textbook outcome.

Results Among 37,707 Medicare beneficiaries, 64.9\% $(n=24,462)$ of patients underwent pancreatic resection while 35.1\% $(n=13,245)$ underwent hepatic resection. Median patient age was 72 years (IQR: 68-77), just over one-half were male $(51.9 \% ; n=19,558)$, and the median CCI was 3 (IQR: 2-8). Cluster analysis revealed five distinct SVI profiles with wide variability in the distribution of SVI subthemes, ranging from 15 (profile 1 IQR: 7-26) to 83 (profile 5 IQR: 66-93). The five profiles were grouped into 3 categories based on median composite SVI: "low vulnerability" (profile 1), "average vulnerability" (profiles 2 and 3), or "high vulnerability" (profiles 4 and 5). The rate of TO ranged from $44.6 \%$ in profile $5(n=4022)$ to $49.2 \%$ in profile $1(n=4836)$. Multivariable analyses comparing patients categorized into the two average SVI profiles revealed that despite having similar composite SVI scores, the risk of adverse postoperative outcomes was not similar. Specifically, patients from profile 5 had lower odds of achieving a TO (OR 0.89, 95\%CI: 0.83-0.95) and higher odds of 90-day mortality (OR 1.29, 95\%CI: 1.15-1.44) versus patients in profile 4.

Conclusion Distinct profiles of SVI subtheme characteristics were independently associated with postoperative outcomes among Medicare beneficiaries undergoing HP surgery, even among patients with similar overall composite SVI scores.
\end{abstract}

Keywords Social vulnerability $\cdot$ Textbook outcome $\cdot$ Hepatopancreatic surgery $\cdot$ Social determinants of health

\section{Introduction}

Social determinants of health (SDH) are increasingly recognized as critical non-medical factors that impact overall health. ${ }^{1,2} \mathrm{SDH}$ represent a complex interplay of elements including socioeconomic status (SES), race, disability, and

Timothy M. Pawlik

tim.pawlik@osumc.edu

1 The Ohio State University Wexner Medical Center, The James Comprehensive Cancer Center, Columbus, OH, USA residential environment that are known to impact both the development and outcomes of medical conditions such as diabetes, ${ }^{3}$ cardiovascular disease, ${ }^{4}$ and even COVID-19. ${ }^{5}$ However, the effects of SDH on surgical outcomes have only more recently been demonstrated. The World Health Organization's Commission on Social Determinants of Health has stressed the importance of recognizing the causes of these disparities and promoting targeted interventions to combat them. ${ }^{6}$ While age, sex, ethnicity/race, SES, and insurance status have previously been associated with surgical 
outcomes, ${ }^{7-10}$ these individual factors do not adequately reflect the complex relationships among SDH.

Social vulnerability index (SVI) is a composite score that encompasses 15 census-tract-level social variables divided among four subthemes: (1) SES, (2) household composition and disability, (3) minority status and language, and (4) housing type and transportation. SVI was initially developed in 2011 to identify communities that might require local or federal support in the event of natural or man-made disasters. ${ }^{11}$ Because multiple social and economic factors are taken into account, SVI has also proven to be a useful surrogate to measure SDH and identify residential areas that are most likely to experience poor health outcomes. ${ }^{12,13}$ Indeed, recent research has noted that patients living in areas with higher composite SVI are more likely to experience worse postoperative outcomes following various surgical procedures including cholecystectomy, colectomy, lung resection, coronary artery bypass grafting, joint replacement, and hepatopancreatic (HP) surgery. ${ }^{14-19}$ However, these studies also demonstrated marked heterogeneity in outcomes even among communities with similar composite SVI. More recently, using cluster analysis, we reported that two of the four subthemes - "SES" and "household composition and disability" - were most strongly associated with poor postoperative outcomes among patients undergoing a wide range of surgical procedures..$^{20,21}$

Hepatic and pancreatic resection are complex procedures that may lead to high rates of postoperative morbidity and prolonged need for recovery. While patients with high SVI may be less likely to receive care at high volume centers, ${ }^{16,17,22}$ which could contribute to worse outcomes, ${ }^{16,17,22,22-24}$ the lack of access to centralized care does not completely explain the discrepancy in post-hepatectomy and post-pancreatectomy outcomes experienced by patients residing in areas of high versus low composite SVI. ${ }^{22,25}$ In order to better understand the impact of SDH on the outcomes of complex HP surgery and identify areas that would most benefit from policy reform, perioperative interventions, and/or community program support, we sought to define the impact of SVI subtheme scores relative to the likelihood of achieving an "optimal" postoperative textbook surgical outcome. We hypothesized that certain SVI subthemes would improve risk stratification among patients undergoing HP surgery who reside in highly distressed areas with poor SDH.

\section{Methods}

\section{Data Source}

Patients were identified from the 2013-2017 100\% Medicare Standard Analytic Files (SAFs), an administrative billing database developed and maintained by the Centers for Medicare and Medicaid Services. ${ }^{26}$ The SAFs contain encounterlevel data on diagnoses and procedures in the form of International Classification of Disease Ninth and Tenth editions (ICD-9/10), as well as patient demographic information and expenditures. Patients who underwent a pancreatectomy or hepatectomy between 2013 and 2017 for any indication, were enrolled in Medicare Parts A and B, were not enrolled in a health maintenance organization, and lived in a county with available data on SVI were included. Supplementary Table 1 lists the ICD-9/10 codes used to identify patients undergoing HP surgery. In instances where a patient underwent more than one procedure of interest between 2013 and 2017 , only the first surgery was analyzed. This study was deemed exempt by the Ohio State University Wexner Medical Center Institutional Review Board.

\section{Independent Variables and Outcomes}

The primary independent variable was the SVI profile. SVI is a composite variable developed and made freely available by the Centers for Disease Control and Prevention. Utilized at the county level, SVI is available as an overall metric, here referred to as "composite SVI" (range: 0-100 with higher values representing more vulnerability); SVI can be divided into four subthemes scores: (1) SES, (2) household composition and disability, (3) minority status and language, and (4) housing type and transportation. To develop the SVI profile, the four subthemes were entered into a cluster analysis using Ward's minimum variance method, as previously described. ${ }^{20}$ Cluster analysis is a form of unsupervised machine learning that produces groups of patients with minimized within-group variability while maximizing between-group variability. Prior to cluster analysis, each of the four subthemes was transformed into percentiles to standardize scaling. The resultant vulnerability profiles were then ordered into hierarchical groups of "low," "average," or "high" vulnerability based on median composite SVI, as previously described. ${ }^{20}$ Secondary independent variables calculated for inclusion in this study were age, gender, racial/ethnic minority status, Charleson Comorbidity Index (CCI), teaching hospital status, emergent versus elective procedures, and hospital volume. Hospital volume was calculated as the total number of HP procedures performed at a particular hospital, identified using the hospital's NPI number.

The primary outcome was "textbook outcome" (TO), a composite outcome defined as the absence of all four component elements: 90-day readmission, complication, extended length of stay (LOS), and 90-day mortality. Secondary outcomes were each of the four individual components of TO. Complications were identified using a previously published and validated list of ICD-9/10 codes. ${ }^{27,28}$ Extended LOS was 
defined as any procedure-specific $\operatorname{LOS}>75$ th percentile. $^{20}$ Readmissions were calculated for readmissions to the same or different hospitals in which the index procedure occurred. Additional details of TO have been outlined previously. ${ }^{29-31}$

\section{Statistical Analysis}

Descriptive statistics were presented as frequency (relative frequency [\%]) for categorical variables and median (25th-75th percentiles [interquartile range, IQR]) for continuous variables. To assess differences in outcomes between SVI profiles after controlling for patient and clinical characteristics, multivariable logistic regression was utilized. All analyses were conducted using SAS v9.4 (SAS Institute, Inc, Cary, NC). Statistical significance was assessed at $\alpha=0.05$.

\section{Results}

\section{Demographic Data}

A total of 37,707 patients who underwent pancreatic or hepatic resection between 2013 and 2017 were included in the study cohort (Table 1). Among all patients, $64.9 \%$ $(n=24,462)$ underwent pancreatic resection while $35.1 \%$ $(n=13,245)$ underwent hepatic resection. Median patient age was 72 years (IQR: 68-77), just over one-half were male $(51.9 \% ; n=19,558)$, and the median CCI was 3 (IQR: 2-8); only approximately one in ten individuals identified as a racial/ethnic minority $(11.7 \% ; n=4416)$. The majority underwent the surgical procedure at a teaching hospital (75.5\%; $n=28,485)$. Most patients $(n=26,540,70.4 \%)$ had a cancer diagnosis as the indication for surgery, with a similar proportion among the different SVI profile groups (range: 69.8 to $71.5 \%$ ). A total of 1563 hospitals performed at least one HP procedure during the study period; the median hospital HP procedural volume was 3 (IQR: 1-16).

\section{SVI and Cluster Analysis}

Cluster analysis revealed five distinct SVI profiles with wide variability in the distribution of SVI subthemes (Table 1 and Fig. 1). These profiles were numbered in ascending order based on median composite SVI, which ranged from 15 (profile 1 IQR: 7-26) to 83 (profile 5 IQR: 66-93). The five profiles were subsequently grouped into 3 categories based on median composite SVI: "low vulnerability" (profile 1), "average vulnerability" (profiles 2 and 3), or "high vulnerability" (profiles 4 and 5).

Despite similar composite SVI, the SVI subtheme scores markedly diverged among individuals categorized into the two "average vulnerability" profiles. Profile 2 had

Table 1 Top half: demographic data stratified by vulnerability cluster profile. Bottom Half: median (and range) composite SVI score and SVI subtheme scores, stratified by vulnerability cluster profile

\begin{tabular}{|c|c|c|c|c|c|c|}
\hline & \multirow[t]{2}{*}{ Total } & \multirow{2}{*}{$\begin{array}{l}\text { Low vulnerability } \\
\text { Profile } 1 \mathrm{~N}=9833\end{array}$} & \multicolumn{2}{|c|}{ Average vulnerability } & \multicolumn{2}{|l|}{ High vulnerability } \\
\hline & & & Profile $2 \mathrm{~N}=6491$ & Profile $3 \mathrm{~N}=6057$ & Profile $4 \mathrm{~N}=6301$ & Profile $5 \mathrm{~N}=9025$ \\
\hline \multicolumn{7}{|l|}{ Procedure } \\
\hline Liver & $13,245(35.1 \%)$ & $3454(35.1 \%)$ & $2272(35 \%)$ & $2131(35.2 \%)$ & $2261(35.9 \%)$ & $3127(34.6 \%)$ \\
\hline Pancreas & $24,462(64.9 \%)$ & $6379(64.9 \%)$ & $4219(65 \%)$ & $3926(64.8 \%)$ & $4040(64.1 \%)$ & $5898(65.4 \%)$ \\
\hline Male gender & $19,558(51.9 \%)$ & $5096(51.8 \%)$ & $3335(51.4 \%)$ & $3201(52.8 \%)$ & $3223(51.2 \%)$ & $4703(52.1 \%)$ \\
\hline Racial/ethnic minority & $4416(11.7 \%)$ & $928(9.4 \%)$ & $777(12.0 \%)$ & $381(6.3 \%)$ & $1179(18.7 \%)$ & $1151(12.8 \%)$ \\
\hline Age (years) & $72(68,77)$ & $72(68,77)$ & $72(68,77)$ & $72(68,76)$ & $72(68,77)$ & $72(68,76)$ \\
\hline $\begin{array}{l}\text { Charlson comorbidity } \\
\text { index }\end{array}$ & $3(2,8)$ & $3(2,8)$ & $3(2,8)$ & $3(2,8)$ & $3(2,8)$ & $3(2,8)$ \\
\hline Teaching hospital & $28,485(75.5 \%)$ & $6852(69.7 \%)$ & $5036(77.6 \%)$ & $4901(80.9 \%)$ & $4466(70.9 \%)$ & $7230(80.1 \%)$ \\
\hline Emergent procedure & $5025(13.3 \%)$ & $1124(11.4 \%)$ & $776(12.0 \%)$ & $882(14.6 \%)$ & $890(14.1 \%)$ & $1353(15.0 \%)$ \\
\hline Cancer Dx & $26,540(70.4 \%)$ & $6896(70.1 \%)$ & $4572(70.4 \%)$ & $4269(70.5 \%)$ & $4506(71.5 \%)$ & $6297(69.8 \%)$ \\
\hline & \multicolumn{6}{|c|}{ Social vulnerability index } \\
\hline Overall SVI & $49(24,74)$ & $15(7,26)$ & $42(25,51)$ & $46(32,61)$ & $74(63,82)$ & $83(66,93)$ \\
\hline Socioeconomic & $49(24,74)$ & $16(7,25)$ & $34(23,43)$ & $62(49,80)$ & $67(56,78)$ & $78(63,92)$ \\
\hline $\begin{array}{l}\text { Household composition } \\
\text { and disability }\end{array}$ & $50(24,74)$ & $26(13,41)$ & $20(5,37)$ & $76(67,88)$ & $41(28,58)$ & $81(72,91)$ \\
\hline $\begin{array}{l}\text { Minority status and } \\
\text { language }\end{array}$ & $50(25,75)$ & $40(20,61)$ & $54(32,71)$ & $19(7,32)$ & $86(77,94)$ & $51(31,70)$ \\
\hline $\begin{array}{l}\text { Housing type and trans- } \\
\text { portation }\end{array}$ & $49(25,74)$ & $18(8,35)$ & $70(57,82)$ & $24(14,33)$ & $77(56,85)$ & $69(54,85)$ \\
\hline
\end{tabular}

SVI, social vulnerability index 


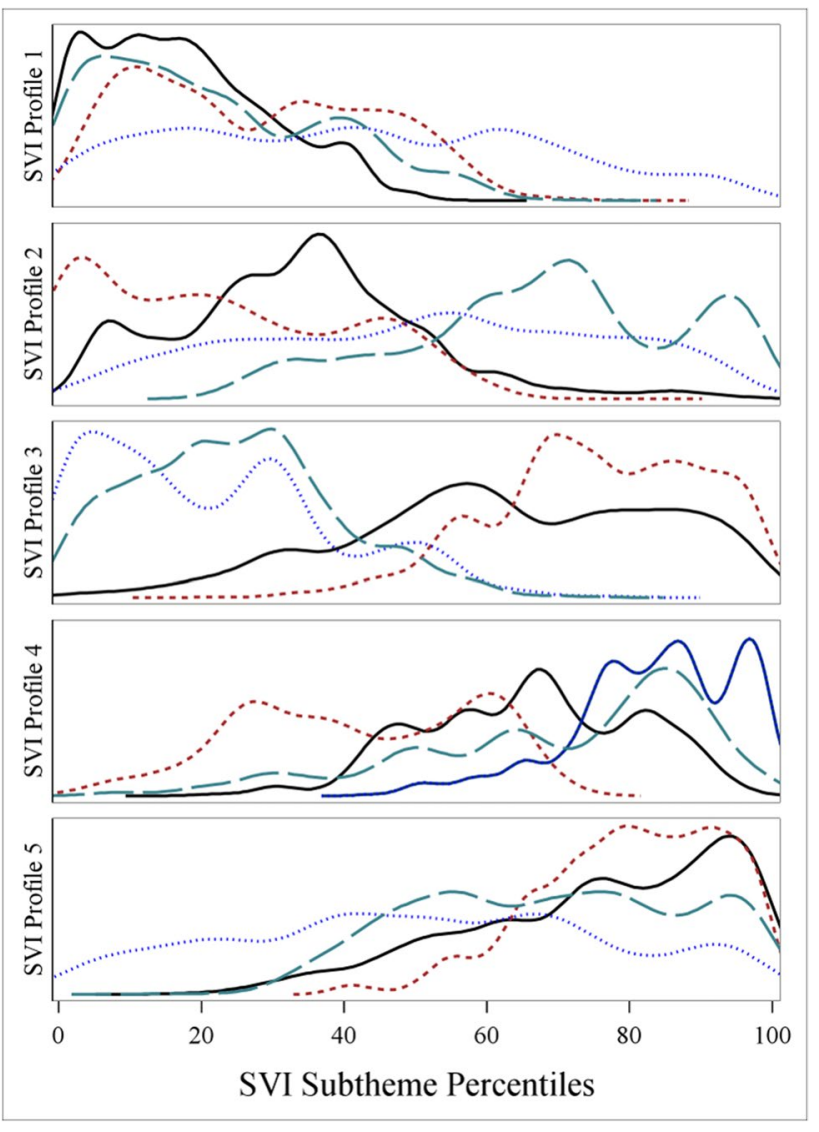

Fig. 1 Line graph depicting the distribution of patient SVI subtheme scores within each SVI profile. SVI subtheme scores are shown as percentiles. Household composition and disability (red dotted line). Socioeconomic Status (black line). Minority Status and Language (blue line). Housing Type and Transportation (green dashed line)

below-average subtheme vulnerability for SES (median: 34; IQR: 23-43) and household composition and disability (median: 20; IQR: 5-37), yet average subtheme vulnerability for minority status and language (median: 54; IQR: 32-71) and above-average subtheme vulnerability for housing type and transportation (median: 70; IQR: 57-82). In contrast, profile 3 had above-average subtheme vulnerability for SES (median: 62; IQR: 49-80) and household composition and disability (median: 76; IQR: 67-88), but below-average subtheme vulnerability for minority status and language (median: 19; IQR: 7-32) and housing type and transportation (median: 24; IQR: 14-33).

Of note, patients who comprised the two high vulnerability profiles had different SVI subtheme score distributions despite similar composite SVI. For example, profile 4 had below-average subtheme vulnerability for household composition and disability (median: 41; IQR: 28-58), yet above-average vulnerability for the other three subthemes: SES (median: 67; IQR: 56-78), minority status and language (median: 86; IQR: 77-94), and housing type and transportation (median: 77; IQR: 56-85). Profile 5 had average subtheme vulnerability for minority status and language (median: 51; IQR: 31-70), but above-average vulnerability for the other 3 subthemes: SES (median: 78; IQR: 63-92), household composition and disability (median: 81; IQR: 72-91), and housing type and transportation (median: 69; IQR: 54-85).

\section{Comparisons of "Low" Vulnerability with Other Vulnerability Profiles}

Bivariate assessments of SVI profiles demonstrated that procedure, gender, age, and CCI distribution was similar across SVI profiles although the distribution of racial/ethnic minority patients ranged from $6.3 \%$ in profile $3(n=381)$ to nearly triple that in profile $4(18.7 \% ; n=1179)$ (Table 1$)$. Of particular note, outcomes varied across SVI profiles (Table 2). The rate of TO ranged from $44.6 \%$ in profile $5(n=4022)$ to $49.2 \%$ in profile $1(n=4836)$. In addition, patients in profile 5 were more likely to experience extended LOS (24.1\% vs. $21.0 \%$ ), a postoperative complication (30.2\% vs. $25.9 \%)$, and mortality within 90 days (10.3\% vs. $7.4 \%)$. Multivariable analyses revealed that patients with an average or high

Table 2 Rates of textbook outcome, 90-day readmission, complication, extended LOS, and 90-day mortality, stratified by vulnerability cluster profile

\begin{tabular}{|c|c|c|c|c|c|c|}
\hline & \multirow[t]{2}{*}{ Total } & \multirow{2}{*}{$\begin{array}{l}\text { Low vulnerability } \\
\text { Profile } 1\end{array}$} & \multicolumn{2}{|c|}{ Average vulnerability } & \multicolumn{2}{|c|}{ High vulnerability } \\
\hline & & & Profile 2 & Profile 3 & Profile 4 & Profile 5 \\
\hline Textbook outcome & $17,815(47.2 \%)$ & $4836(49.2 \%)$ & $3132(48.3 \%)$ & $2827(46.7 \%)$ & $2998(47.6 \%)$ & $4022(44.6 \%)$ \\
\hline 90-day readmission & $11,142(29.5 \%)$ & $2888(29.4 \%)$ & $1913(29.5 \%)$ & $1761(29.1 \%)$ & $1802(28.6 \%)$ & $2778(30.8 \%)$ \\
\hline Complication & $10,595(28.1 \%)$ & $2543(25.9 \%)$ & $1729(26.6 \%)$ & $1791(29.6 \%)$ & $1807(28.7 \%)$ & $2725(30.2 \%)$ \\
\hline Extended LOS & $8515(22.6 \%)$ & $2062(21.0 \%)$ & $1418(21.8 \%)$ & $1391(23.0 \%)$ & $1468(23.3 \%)$ & $2176(24.1 \%)$ \\
\hline 90-day mortality & $3256(8.6 \%)$ & $729(7.4 \%)$ & $522(8.0 \%)$ & $561(9.3 \%)$ & $518(8.2 \%)$ & $926(10.3 \%)$ \\
\hline Readmission to different hosp. & $3490(9.3 \%)$ & $870(8.9 \%)$ & $552(8.5 \%)$ & $599(9.9 \%)$ & $553(8.8 \%)$ & $916(10.2 \%)$ \\
\hline
\end{tabular}

$L O S$, length of stay 
vulnerability profile had worse outcomes compared with patients who had a low vulnerability profile (Table 3 ).

\section{Comparisons of Vulnerability Profiles}

Multivariable analyses comparing patients categorized into the two average SVI profiles revealed that despite having similar composite SVI scores, the risk of adverse postoperative outcomes was not similar (Table 4). Patients in profile 3 had higher odds of experiencing a complication (OR 1.13, 95\%CI: $1.04-1.23$ ) and 90-day mortality (OR 1.14, 95\% CI: 1.01-1.30) compared with patients in SVI profile 2. Similarly, despite comparable composite SVI scores, patients who were categorized into the two high SVI profiles had different outcomes. Specifically, patients from profile 5 had lower odds of achieving a TO (OR 0.89, 95\%CI: 0.83-0.95) and higher odds of 90-day mortality (OR 1.29, 95\%CI: $1.15-1.44)$ versus patients in profile 4 .

\section{Discussion}

SVI can be used as a measure of SDH to identify patient groups who may be most at risk for poor postoperative outcomes following a range of complex surgical procedures. ${ }^{14-18}$ The current study elucidated the association between SVI subthemes and outcomes of patients undergoing hepatic and pancreas resection, two particularly highrisk surgeries. Using cluster analysis, we identified 5 distinct subtheme profiles and noted that SES and household composition and disability were the subthemes most strongly associated with poor postoperative outcomes. By identifying subthemes of particular importance, these data may improve stratification for individuals undergoing complex HPB surgery, help deliver resources to facilitate patient care, improve communication, as well as inform greater care coordination with nurse navigator follow-up.

A novel aspect of our study was the use of cluster analysis, which demonstrated two groups with average composite SVI and two groups with high composite SVI that each
Table 4 Multivariable analysis comparing outcomes in vulnerability cluster profile 3 compared to profile 2 , and in vulnerability cluster profile 5 compared with profile 4

\begin{tabular}{lll}
\hline & $\begin{array}{l}\text { Average vulnerability } \\
\text { Profile 2 vs. 3 }\end{array}$ & $\begin{array}{l}\text { High vulnerability } \\
\text { Profile 4 vs. 5 }\end{array}$ \\
\hline Textbook outcome & $0.96(0.90,1.04)$ & $\mathbf{0 . 8 9}(\mathbf{0 . 8 3}, \mathbf{0 . 9 5})$ \\
90-day readmission & $0.97(0.90,1.05)$ & $1.01(0.93,1.09)$ \\
Complication & $\mathbf{1 . 1 3}(\mathbf{1 . 0 4}, \mathbf{1 . 2 3})$ & $1.05(0.97,1.14)$ \\
Extended LOS & $1.02(0.94,1.12)$ & $1.04(0.96,1.13)$ \\
90-day mortality & $\mathbf{1 . 1 4}(\mathbf{1 . 0 1}, \mathbf{1 . 3 0})$ & $\mathbf{1 . 2 9}(\mathbf{1 . 1 5}, \mathbf{1 . 4 4})$ \\
\hline
\end{tabular}

Analyses controlled for patient age, race/ethnicity, gender, CCI as well as the procedure type, emergent/elective status, and hospital volume. Odds ratio (95\% confidence interval). Bolded results are significant $(p \leq 0.05)$

$L O S$, length of stay

had a unique SVI subtheme profile. Profiles 3 and 5 had higher subtheme scores for SES and household composition and disability, but lower subtheme scores for minority status and language and housing type and transportation compared with profiles 2 and 4, respectively. These findings support the notion that SES and household composition and disability appear to be the subthemes most responsible for worse surgical outcomes associated with increased composite SVI. These findings remained even after controlling for age, sex, race/ethnicity, CCI, teaching hospital status, and the emergent versus the elective status of the procedure. SES is generally considered to be one of the strongest predictors of postoperative mortality. ${ }^{32,33}$ Similarly, the household composition and disability subtheme encompasses multiple factors that are associated with poor surgical outcomes, such as patients at the extremes of age $\mathrm{e}^{34,35}$ or those individuals who have a disability. ${ }^{36,37}$

Our findings are consistent with the results of other recent studies on SVI subtheme analysis, which noted that SES and household composition and disability were the subthemes most often associated with worse postoperative outcomes among patients undergoing lung resection, coronary artery bypass grafting, abdominal aortic aneurysm repair, and
Table 3 Multivariable analysis comparing postoperative outcomes in each vulnerability cluster profile compared to profile 1

\begin{tabular}{lllll}
\hline & Profile 2 & Profile 3 & Profile 4 & Profile 5 \\
\hline Textbook outcome & $0.97(0.91,1.03)$ & $\mathbf{0 . 9 3}(\mathbf{0 . 8 7}, \mathbf{0 . 9 9})$ & $0.96(0.90,1.02)$ & $\mathbf{0 . 8 5}(\mathbf{0 . 8 0 , 0 . 9 1})$ \\
90-day readmission & $1.01(0.94,1.08)$ & $0.97(0.91,1.05)$ & $0.97(0.90,1.04)$ & $1.06(0.99,1.13)$ \\
Complication & $1.03(0.96,1.11)$ & $\mathbf{1 . 1 7}(\mathbf{1 . 0 9 , 1 . 2 6})$ & $\mathbf{1 . 1 1}(\mathbf{1 . 0 4}, \mathbf{1 . 2 0})$ & $\mathbf{1 . 1 9}(\mathbf{1 . 1 2 , 1 . 2 7})$ \\
Extended LOS & $1.04(0.96,1.13)$ & $1.07(0.98,1.16)$ & $1.08(0.99,1.17)$ & $\mathbf{1 . 1 2}(\mathbf{1 . 0 4 , 1 . 2 1})$ \\
90-day mortality & $1.08(0.96,1.22)$ & $\mathbf{1 . 2 4}(\mathbf{1 . 1 0 , 1 . 3 9})$ & $1.08(0.96,1.22)$ & $\mathbf{1 . 3 9}(\mathbf{1 . 2 5}, \mathbf{1 . 5 4})$ \\
\hline
\end{tabular}

Analyses controlled for patient age, race/ethnicity, gender, CCI as well as the procedure type, emergent/ elective status, and hospital volume. Reported as odds ratio ( $95 \%$ confidence interval). Bolded results are significant $(p \leq 0.05)$

$L O S$, length of stay 
colectomy. ${ }^{20,21}$ Future research should evaluate whether subtheme characteristics are more prognostic than individual component elements of SDH or whether composite SVI scores can better identify patients at risk for adverse outcomes. Data from the current study would strongly suggest the former rather than the latter. In particular, we observed that despite patients in profile 3 (average SVI) having a lower composite SVI than individuals in profile 4 (high SVI), profile 3 was associated with lower rates of TO, and higher rates of 90-day readmission, complications, and mortality. Thus, while composite SVI was associated with postoperative outcomes following HP, specific SDH subtheme characteristics such as SES and household composition and disability appeared to be the most important drivers of postoperative outcomes.

The current study therefore served to emphasize the important role of SVI subtheme analysis. While there are several SDH screening tools that can be used to identify at-risk patients, these tools mostly focus on ascertaining general patient needs. ${ }^{38-40}$ Subtheme analysis may allow healthcare teams to identify patient needs more accurately as well as highlight why patients may be at particular risk for poor surgical outcomes, thereby allowing delivery of targeted support services. In particular, identification of which SDH elements place patients at risk may help aim interventions at ensuring stable housing after surgery, availability of transportation to health care appointments, or access to nutritious food (e.g., the supplemental nutrition assistance program, known as SNAP). Helping patients access these types of community programs can, in turn, improve health outcomes. ${ }^{41,42}$ For example, improvement in chronic health conditions among low SES families can be improved through housing support. ${ }^{43}$ At the community level, subtheme analysis highlights those social problems most in need of being targeted for social programs and policies.

The current study had several limitations. As the data were derived from the Medicare administrative billing database, we were limited to utilizing ICD-9 and ICD-10 codes. Therefore, it was possible that records on demographic factors, comorbidities, or complications were incomplete. Prior studies have demonstrated, however, that miscoding of surgical procedures was uncommon and therefore this potential shortcoming was unlikely to impact the results. ${ }^{44}$ Only patients enrolled in Medicare were included in the analytic cohort in order to decrease the confounding effects of insurance status on outcomes. As such, the results may not be applicable to patients not on Medicare (e.g., younger, privately insured); however, the SAFs provide a comprehensive picture of healthcare utilization and surgical outcomes for a national sample while also providing information on patient residence - none of which is available in other, administrative billing databases that contain younger, privately insured patients (e.g., MarketScan). Furthermore, there was a smaller proportion of people who identified as racial and ethnic minorities in this sample population compared with the United States population. ${ }^{45}$ It was possible that this disparity was actually representative of the disproportionate access to surgical care experienced by patients of racial and ethnic minorities. ${ }^{46,47}$ In addition, it is important to note that SVI was calculated at a census-tract level and therefore was not necessarily reflective of individual patient-level SDH.

In conclusion, distinct profiles of SVI subtheme characteristics were independently associated with postoperative outcomes among Medicare beneficiaries undergoing HP surgery, even among patients with similar overall composite SVI scores. In particular, the subthemes of SES and household composition and disability were the biggest drivers in determining postoperative surgical outcomes. Future research is needed to determine whether identifying and targeting interventions aimed at certain characteristics of SDH and social vulnerability can improve outcomes among patients undergoing complex surgery such as liver or pancreas resection.

Supplementary Information The online version contains supplementary material available at https://doi.org/10.1007/s11605-022-05245-9.

\section{References}

1. Organization, W.H. Social Determinants of Health. 08/17/2021]; Available from: https://www.who.int/health-topics/social-deter minants-of-health\#tab=tab_1.

2. Braveman, P. and L. Gottlieb, The social determinants of health: it's time to consider the causes of the causes. Public Health Rep, 2014. 129 Suppl 2: p. 19-31.

3. Walker, R.J., et al., Impact of social determinants of health on outcomes for type 2 diabetes: a systematic review. Endocrine, 2014. 47(1): p. 29-48.

4. Arnett, D.K., et al., 2019 ACC/AHA Guideline on the Primary Prevention of Cardiovascular Disease: A Report of the American College of Cardiology/American Heart Association Task Force on Clinical Practice Guidelines. Circulation, 2019. 140(11): p. e596-e646.

5. Singu, S., et al., Impact of social determinants of health on the emerging COVID-19 pandemic in the United States. Front Public Health, 2020. 8: p. 406.

6. Marmot, M., et al., Closing the gap in a generation: health equity through action on the social determinants of health. Lancet, 2008. 372(9650): p. 1661-9.

7. Schneider, E.B., et al., Differential association of race with treatment and outcomes in Medicare patients undergoing diverticulitis surgery. Arch Surg, 2011. 146(11): p. 1272-6.

8. Melvan, J.N., et al., Nonclinical factors associated with 30-day mortality after lung cancer resection: an analysis of 215,000 patients using the National Cancer Data Base. J Am Coll Surg, 2015. 221(2): p. 550-63.

9. Rozental, O., et al., Disparities in mortality after abdominal aortic aneurysm repair are linked to insurance status. J Vasc Surg, 2020. 72(5): p. 1691-1700 e5. 
10. Park, H.S., et al., Social determinants of health and their impact on postcolectomy surgery readmissions: a multistate analysis, 2009-2014. J Comp Eff Res, 2019. 8(16): p. 1365-1379.

11. Flanagan BE, G.E., Hallisey EJ, Heitgerd JL, Lewis B, A social vulnerability index for disaster management. Journal of Homeland Security and Emergency Management, 2011. 8(1).

12. Biggs, E.N., et al., The relationship between social vulnerability and COVID-19 incidence among Louisiana census tracts. Front Public Health, 2020. 8: p. 617976.

13. (ATSDR), A.f.T.S.a.D.R. CDC/ATSDR social vulnerability index. 2021 August 28, 2021]; Available from: https://www.atsdr.cdc. gov/placeandhealth/svi/index.html.

14. Hyer, J.M., et al., High social vulnerability and "textbook outcomes" after cancer operation. J Am Coll Surg, 2021. 232(4): p. 351-359.

15. Diaz, A., et al., County-level social vulnerability is associated with worse surgical outcomes especially among minority patients. Ann Surg, 2020. Publish Ahead of Print.

16. Diaz, A., et al., Association of neighborhood characteristics with utilization of high-volume hospitals among patients undergoing high-risk cancer surgery. Ann Surg Oncol, 2021. 28(2): p. 617-631.

17. Azap, R.A., et al., The association of neighborhood social vulnerability with surgical textbook outcomes among patients undergoing hepatopancreatic surgery. Surgery, 2020. 168(5): p. 868-875.

18. Diaz, A., et al., Intersection of social vulnerability and residential diversity: postoperative outcomes following resection of lung and colon cancer. J Surg Oncol, 2021.

19. Carmichael, H., et al., Using the social vulnerability index to examine local disparities in emergent and elective cholecystectomy. J Surg Res, 2019. 243: p. 160-164.

20. Paro, A., et al., Profiles in social vulnerability: the association of social determinants of health with postoperative surgical outcomes. Surgery, 2021.

21. Diaz, A., et al., The impact of social vulnerability subthemes on postoperative outcomes differs by racial/ethnic minority status. Am J Surg, 2021.

22. Diaz, A., et al., Association of social vulnerability with the use of high-volume and magnet recognition hospitals for hepatopancreatic cancer surgery. Surgery, 2021. 170(2): p. 571-578.

23. Song, Y., et al., National trends in centralization and perioperative outcomes of complex operations for cancer. Surgery, 2019. 166(5): p. 800-811.

24. Coon, C., et al., Primary liver cancer: an NCDB analysis of overall survival and margins after hepatectomy. Ann Surg Oncol, 2020. 27(4): p. 1156-1163.

25. Hyer, J.M., et al., Patient social vulnerability and hospital community racial/ethnic integration: do all patients undergoing pancreatectomy receive the same care across hospitals? Ann Surg, 2021. 274(3): p. 508-515.

26. Services, C.f.M.a.M., Limited data set (LDS) files.

27. Quan, H., et al., Coding algorithms for defining comorbidities in ICD-9-CM and ICD-10 administrative data. Med Care, 2005. 43(11): p. 1130-9.

28. Iezzoni, L.I., et al., Identifying complications of care using administrative data. Med Care, 1994. 32(7): p. 700-15.

29. Merath, K., et al., Textbook outcomes among medicare patients undergoing hepatopancreatic surgery. Ann Surg, 2020. 271(6): p. 1116-1123.

30. Mehta, R., et al., Dedicated cancer centers are more likely to achieve a textbook outcome following hepatopancreatic surgery. Ann Surg Oncol, 2020. 27(6): p. 1889-1897.
31. Mehta, R., et al., Comparing textbook outcomes among patients undergoing surgery for cancer at U. S. News \& World Report ranked hospitals. J Surg Oncol, 2020. 121(6): p. 927-935.

32. Reames, B.N., et al., Socioeconomic disparities in mortality after cancer surgery: failure to rescue. JAMA Surg, 2014. 149(5): p. 475-81.

33. Jerath, A., et al., Socioeconomic status and days alive and out of hospital after major elective noncardiac surgery: a populationbased cohort study. Anesthesiology, 2020. 132(4): p. 713-722.

34. Turrentine, F.E., et al., Surgical risk factors, morbidity, and mortality in elderly patients. J Am Coll Surg, 2006. 203(6): p. 865-77.

35. Ramanathan, R., et al., Age-related risks of complications after distal pancreatectomy for neuroendocrine and cystic neoplasms. HPB (Oxford), 2019. 21(7): p. 810-817.

36. Ghani, M., et al., Vascular surgery receipt and outcomes for people with serious mental illnesses: retrospective cohort study using a large mental healthcare database in South London. J Psychosom Res, 2021. 147: p. 110511.

37. Lin, J.A., et al., Postoperative adverse outcomes in intellectually disabled surgical patients: a nationwide population-based study. PLoS One, 2011. 6(10): p. e26977.

38. Buitron de la Vega, P., et al., Implementing an EHR-based screening and referral system to address social determinants of health in primary care. Med Care, 2019. 57 Suppl 6 Suppl 2: p. S133-S139.

39. Thomas-Henkel, C., Schulman, Meryl, Screening for social determinants of health in populations with complex needs: implementation considerations. 2017, Center for Health Care Strategies.

40. Armstrong Brown, J., Berzin O, Clayton M, Cluff L, Derzon J, Evans L, Farrell K, Fraze T, Haber S, Hessler D, Husick C, Liu E, Parish W, Payne J, Renaud J, Rojas Smith L, Stockdale H, Vincent A, Accountable Health Communities (AHC) Model Evaluation First Evaluation Report. 2020.

41. Berkowitz, S.A., et al., Addressing unmet basic resource needs as part of chronic cardiometabolic disease management. JAMA Intern Med, 2017. 177(2): p. 244-252.

42. Gottlieb, L.M., et al., Effects of social needs screening and inperson service navigation on child health: a randomized clinical trial. JAMA Pediatr, 2016. 170(11): p. e162521.

43. Ludwig, J., et al., Neighborhoods, obesity, and diabetes - a randomized social experiment. N Engl J Med, 2011. 365(16): p. 1509-19.

44. Nathan, H. and T.M. Pawlik, Limitations of claims and registry data in surgical oncology research. Ann Surg Oncol, 2008. 15(2): p. 415-23.

45. Bureau, U.S.C., United States Census. 2019.

46 Haider, A.H., et al., Racial disparities in surgical care and outcomes in the United States: a comprehensive review of patient, provider, and systemic factors. J Am Coll Surg, 2013. 216(3): p. 482-92 e12.

47. Salehi, O., et al., Race, age, gender, and insurance status: a comparative analysis of access to and quality of gastrointestinal cancer care. J Gastrointest Surg, 2021. 25(8): p. 2152-2162.

Publisher's Note Springer Nature remains neutral with regard to jurisdictional claims in published maps and institutional affiliations. 